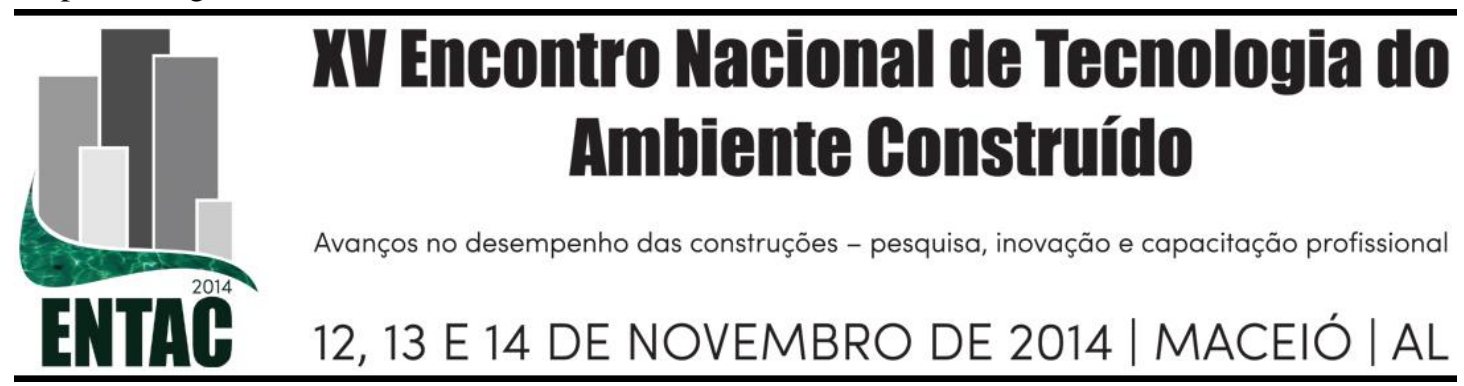

\title{
TECNOLOGIA DE ILUMINAÇÃO LED x FLUORESCENTE T5: UM ESTUDO DE EFICIÊNCIA ENERGÉTICA APLICADO EM LOJA DE CONVENIÊNCIA DE POSTO DE SERVIÇO
}

\author{
DI TRAPANO, Patrizia (1); DE BENEDETTO, Gisele Saveriano (2) \\ (1)AMBEE/FAU/UFRJ, e-mail: patrizia@loggia.arq.br (2) IPIRANGA, email: giselel@ipiranga.com.br
}

\begin{abstract}
RESUMO
O objetivo desse artigo é apresentar os ganhos energéticos obtidos através da mudança de tecnologia de iluminação artificial, e sua integração com a iluminação natural, somada a revisão do nível médio de iluminância de 500 lux para 400 lux, nas lojas de conveniência am/pm dos Postos Ecoeficientes Ipiranga, com base no IES - Illuminating Engineering Society (2011). A tecnologia de iluminação fluorescente T5 $(4 \mathrm{x} 14 \mathrm{~W})+$ PAR vapor metálico $35 \mathrm{~W}$ foi substituída para panel flat LED 40W + PAR LED 12W. A metodologia desenvolveu-se através do estudo de caso realizado na loja am/pm localizada em São Cristovão, no Rio de Janeiro. Foram elaboradas 4 situações de projeto, nas quais foram realizadas medições de iluminância e de consumo de energia durante uma semana em cada uma das situações. Para a realização das medições de iluminância, a norma adotada foi a ABNT NBR ISO/CIE 8995-1 (2013). Foram realizadas simulações com o software AGI-32 para comparação com os resultados das medições, e também elaborados gráficos de consumo em $\mathrm{kWh}$ para cada semana de medição. Como resultado, o estudo mostrou redução de aproximadamente $57 \%$ no consumo de energia elétrica com iluminação, ocorrida com a mudança da tecnologia para LED associada a nova proposta de iluminância. Outra importante constatação foi a redução do DPI (densidade de potência de iluminação) para $9 \mathrm{~W} / \mathrm{m}^{2}$. Este estudo fez parte de um projeto de Pesquisa e Desenvolvimento, com o apoio da FINEP e parceria com universidades, desenvolvido pela Ipiranga Produtos de Petróleo S. A., com foco na evolução da tecnologia LED aplicada na sua rede de postos de serviço. Com base nesses resultados, a iluminação com LED tem sido implementada nas lojas como um item relevante do Posto Ecoeficiente Ipiranga, resultando numa importante contribuição para redução do consumo de energia.
\end{abstract}

Palavras-chave: iluminação, eficiência energética, LED.

\begin{abstract}
The propose of this paper is to present the energy gains obtained through the change of lighting technology and its integration with the natural light, added to the change of illuminance in the am/pm convenience stores of Ipiranga Eco-Efficient Service Station, that includes the revision of the medium level of illuminance of 500 lux for 400 lux as reference level, based on the IES - Illuminating Engineering Society (2011). The fluorescent lighting technology T5(4x14W) + metal halide PAR 35W was replaced for panel flat LED $40 \mathrm{~W}+$ PAR LED $12 \mathrm{~W}$. The methodology developed was the case study in the am/pm convenience store located in São Cristovão, RJ. For this study four design situations were elaborated, in which illuminance measurements and power consumption were performed during one week in each situation. To carry out the measurements of illuminance, the standard adopted was the ABNT NBR ISO/CIE 8995-1 (2013). Simulations were performed with the AGI-32 software to be compared with the results of the measurements. Graphics of energy consumption in kWh were prepared for each week. As a result, the study showed about $57 \%$ reduction in electric power consumption, which occurred due to a change from LEDs technology added to the change of illuminance. Another important finding was the significant decrease of the LPD (lighting power density) to $9 \mathrm{~W} / \mathrm{m} 2$. This study was part of a research and development project on artificial lighting and LED technology on the Ipiranga Service Station chain and
\end{abstract}


it was an important contribution to energy consumption reduction. Based on these results, the LEDs technology has been implemented in the am/pm convenience stores as an important item of the Ipiranga Eco-Efficient Service Stations.

Keywords: lighting, energy efficiency, LED

\section{INTRODUÇÃO}

A Ipiranga Produtos de Petróleo S.A. conduz um amplo projeto de inovação e pesquisa, com o apoio da FINEP e parceria com universidades, com foco na evolução das tecnologias aplicadas na sua rede de Postos Ecoeficientes. O objetivo desse artigo é apresentar os ganhos energéticos obtidos através da mudança de tecnologia de iluminação artificial, e sua integração com a iluminação natural, uma vez que as lojas $\mathrm{am} / \mathrm{pm}$ apresentam domus para aproveitamento da luz natural em seus projetos, envolvendo sensores de controle de iluminação.

A mudança de tecnologia dos Postos Ecoeficientes Ipiranga não se caracterizou como um retrofit, substituindo a atual iluminação fluorescente T5 $(4 \times 14 \mathrm{~W})+$ PAR vapor metálico $35 \mathrm{~W}$ por equipamentos com LEDs. Por se tratar de um projeto de inovação, desenvolveu-se um estudo do projeto luminotécnico atual das lojas de conveniência am/pm, que incluiu a revisão do nível mínimo de iluminância de 500 lux para 400 lux, com base no IES - Illuminating Engineering Society (2011), e a realização do levantamento de iluminâncias em diversas lojas am/pm, que embasaram a pesquisa.

Com relação a substituição da tecnologia fluorescente por LED, a preocupação da Ipiranga era trabalhar com fornecedores que considerassem aspectos técnicos relevantes dos produtos como a potência, fluxo luminoso, temperatura de cor, eficiência $(\mathrm{lm} / \mathrm{W})$, fotometria acreditada em laboratório, garantia, IP (grau de proteção das luminárias). Esse estudo possibilitou à Ipiranga a homologação de fornecedores com maior confiabilidade no resultado luminotécnico do projeto.

A metodologia desenvolveu-se na forma de estudo de caso da loja am/pm situada no Posto Ipiranga Matriz em São Cristóvão, Rio de Janeiro. Nesta loja, foi desenvolvido o projeto de iluminação, com o apoio de simulações computacionais feitas através do software AGI-32, passando-se à fase de protótipo, onde as soluções foram aplicadas para avaliação qualitativa e medição.

O objetivo das medições em 4 etapas foi, além de identificar a economia de energia proveniente da troca de tecnologia e partido de projeto, conhecer a real contribuição da integração da luz artificial com a natural. Os resultados das medições foram comparados com as simulações feitas da etapa de projeto. Os resultados das medições de consumo nos circuitos de iluminação da loja, foram transformados em gráficos de $\mathrm{kWh}$ para cada semana de medição, nas 4 situações propostas.

- Situação 1 e 2 com lâmpadas fluorescentes T5 (4x14W) e PAR vapor metálico 35W, com sensores de iluminância desligados (situação 1) e ligados (situação 2) configurando o projeto padrão atual da loja;

- Situação 3 e 4 com panel flat em LED 40W e PAR 30 em LED 12W com sensores de iluminância desligados (situação 3) e ligados (situação 4) - de acordo com o novo projeto proposto. 


\section{FUNDAMENTAÇÃO}

\subsection{Parâmetros de projeto}

Para as lojas de conveniência am/pm, a recomendação do IES - Illuminating Engineering Society (p.34.8 - p.34.12, 2011) para iluminâncias horizontais na área de loja (food market) é de 500 lux (Figura 1). Como as lojas am/pm possuem também atividade de food service, o IES (p.22.18, 2011) recomenda para essas áreas iluminâncias horizontais de 200 lux (Figura 2).

Evoluindo nas pesquisa, constatou-se durante as medições realizadas em diversas lojas am/pm, que a média de iluminância para luz geral (com a iluminação de destaque deligada) variava entre 250 a $400 \mathrm{lux}$, e as lojas não era consideradas escuras. Como exemplo, a medição realizada na loja am $/ \mathrm{pm}$, estudo de caso desse artigo, no dia 27/03/2012 à noite, onde as lâmpadas de destaque vapor metálico PAR 30/35W foram desligadas, obteve-se média de iluminância de 425 lux. A medição realizada no dia 19/09/2013 à noite, com todo o sistema ligado, resultou na média de 647lux. Constatouse que existe contribuição significativa na iluminância devido a utilização das lâmpadas PAR metálicas de 35Wpara iluminação de destaque, levando à loja a índice de iluminância maior do que a média de 500 lux. Este fato resulta também em maior consumo de energia.

Com base nas observações do IES (2011), que estabelece iluminâncias entre 200lux (food service) a 500 lux (grocery/supermarket), e devido a coexistência dessas atividades no mesmo espaço, somadas as medições realizadas nas lojas am/pm, que variaram entre 250 a 400 lux, foi proposto trabalhar com iluminância média de 400 lux, incluindo luz geral e de destaque, trocando ambas as fontes para LED.

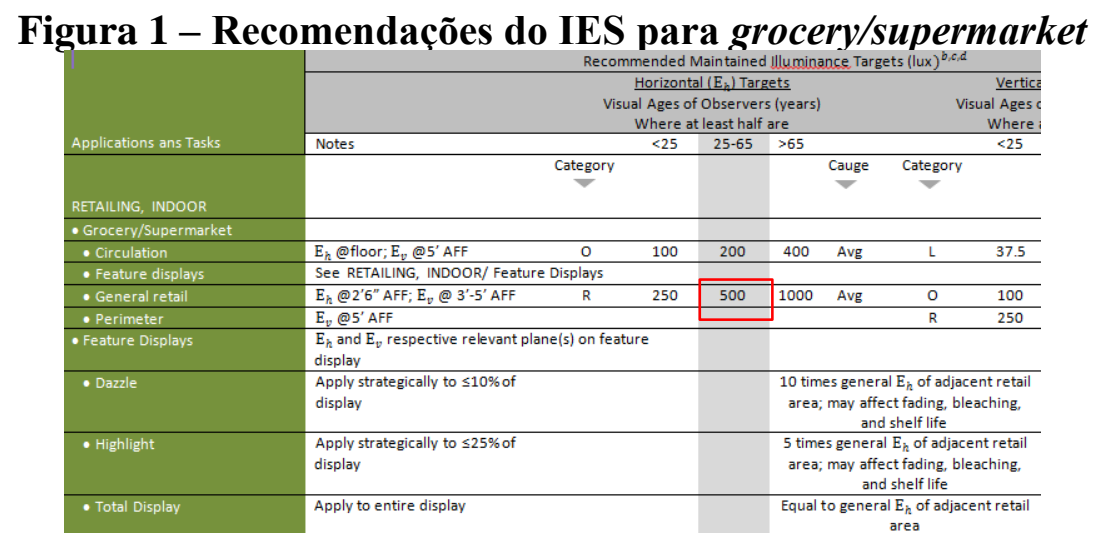

Fonte: (IES, p.34.8-p.34.12, 2011)

Figura 2 - Recomendações do IES para food service

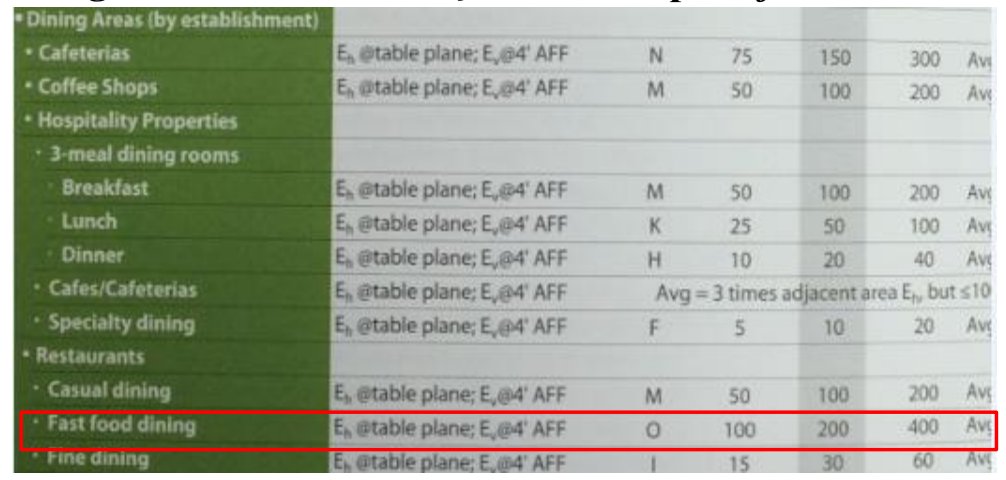

Fonte: (IES, p.22.18, 2011) 
Sobre temperatura de cor é importante ressaltar que, segundo recomendações do IES (2011), para iluminâncias $\leq 500$ lux a aparência de cor deverá ser quente ou intermediária. Isto significa estar entre $3500 \mathrm{~K}$ a $4000 \mathrm{~K}$.

Para a loja am/pm, segundo a ASHRAE 90-1 (2010), o DPI (densidade de potência de iluminação) seria de $18.10 \mathrm{~W} / \mathrm{m}^{2}$. A média das medições realizadas em postos com lâmpadas fluorescentes T5 (4x14W) e PAR vapor metálico $35 \mathrm{~W}$ foi de $12.73 \mathrm{~W} / \mathrm{m}^{2}$, para iluminação geral, sem considerar a iluminação de destaque. Devido as mudanças de parâmetros e de tecnologia, o DPI passou para $9 \mathrm{~W} / \mathrm{m}^{2}$, incluindo iluminação geral e de destaque.

\subsection{Sensores e regulagens}

Com relação aos sensores de controle da iluminação, pode-se dizer que, segundo o IES (2011), a separação de grupos de luminárias em seções controladas por diferentes sensores, torna o sistema mais eficiente, sendo recomendada a instalação de mais sensores ligados as seções paralelas a fonte de luz natural. Este procedimento permite ao sistema de automação operar em diferentes níveis de iluminância compensada pela incidência da luz natural.

Os sensores disponibilizados no mercado apresentam distintos protocolos, sendo os mais usados o de padrão analógico, denominado $1-10 \mathrm{~V}$ e o padrão digital, denominado DALI. O padrão analógico tem a vantagem de se integrar com os reatores dimerizáveis mais comuns de mercado, são relativamente fáceis de encontrar e instalar, porém são pouco versáteis em se tratando de instalação (tem limite de número de luminárias e distância) e são muito imprecisos para calibrar, caso se deseje uma iluminância diferente no nível de referência estabelecido de 500 lux. Por outro lado, o padrão DALI permite que os sensores sejam configurados remotamente, através de uma interface feita pelo computador. Outra vantagem é a precisão e a capacidade de endereçamento digital, que permite ao usuário a programação de linhas, colunas ou até mesmo individualizar a dimerização de cada luminária.

Os sensores instalados na loja matriz foram do fabricante PHILIPS, MicroLuxsense LRL 1220/10, protocolo 1-10V, com o nível de referência de 500 lux. Para alteração do nível de 500 lux para 400 lux (nova proposta de iluminância da loja), foi seguido o procedimento de instalação e ajuste dos sensores MicroLuxsense conforme as instruções fornecidas pelo fabricante.

\section{DESENVOLVIMENTO DA METODOLOGIA}

\subsection{Situação 1 e 2 - Fluorescente T5 $(4 \times 14 W)$ + PAR vapor metálico 35W - sensores desligados (1) e ligados (2)}

As situações 1 e 2 apresentam em sua iluminação geral 23 luminárias do tipo embutido com 4 fluorescentes T5 x 14W, distribuídas em fileiras. Além destas, existem luminárias pontuais para atender a padaria, o balcão caixa e a vitrine da loja com lâmpadas vapor metálico CDMR PAR 30 de 35W (Figura 3). Esta loja é dotada de 4 domus para iluminação natural. No projeto existente na loja, as luminárias fluorescentes estavam ligadas somente a um sensor, com o objetivo de monitorar o nível de iluminância de acordo com a incidência de luz natural proveniente dos domus.

Para atender as recomendações do IES (2011), foram instalados 4 sensores, assinalados em vermelho (Figura 3), ligados a 4 grupos de seções de luminárias com lâmpadas T5 
4x14W (M/L/J/D). As luminárias pontuais não foram ligadas a sensores pelo fato de não serem dimerizadas. Nesta situação, foram realizadas duas semanas de medições de consumo de energia: a primeira com os sensores desligados e a segunda com os sensores ligados. Para o levantamento do consumo de energia elétrica, foi utilizado um medidor eletrônico digital portátil, registrando medições com intervalo de integração de 30 minutos, durante um período de 7 dias. Para realização das medições de iluminâncias, foram consideradas todas as lâmpadas acesas (iluminação geral + pontuais), seguindo a metodologia da malha de cálculo para projeto do sistema de iluminação da Norma ABNT NBR ISO/CIE 8995-1 (2013).

Figura 3 - Planta de teto da situação 1 e 2: fluorescente T5 4X14W + VM 35W com a marcação dos sensores

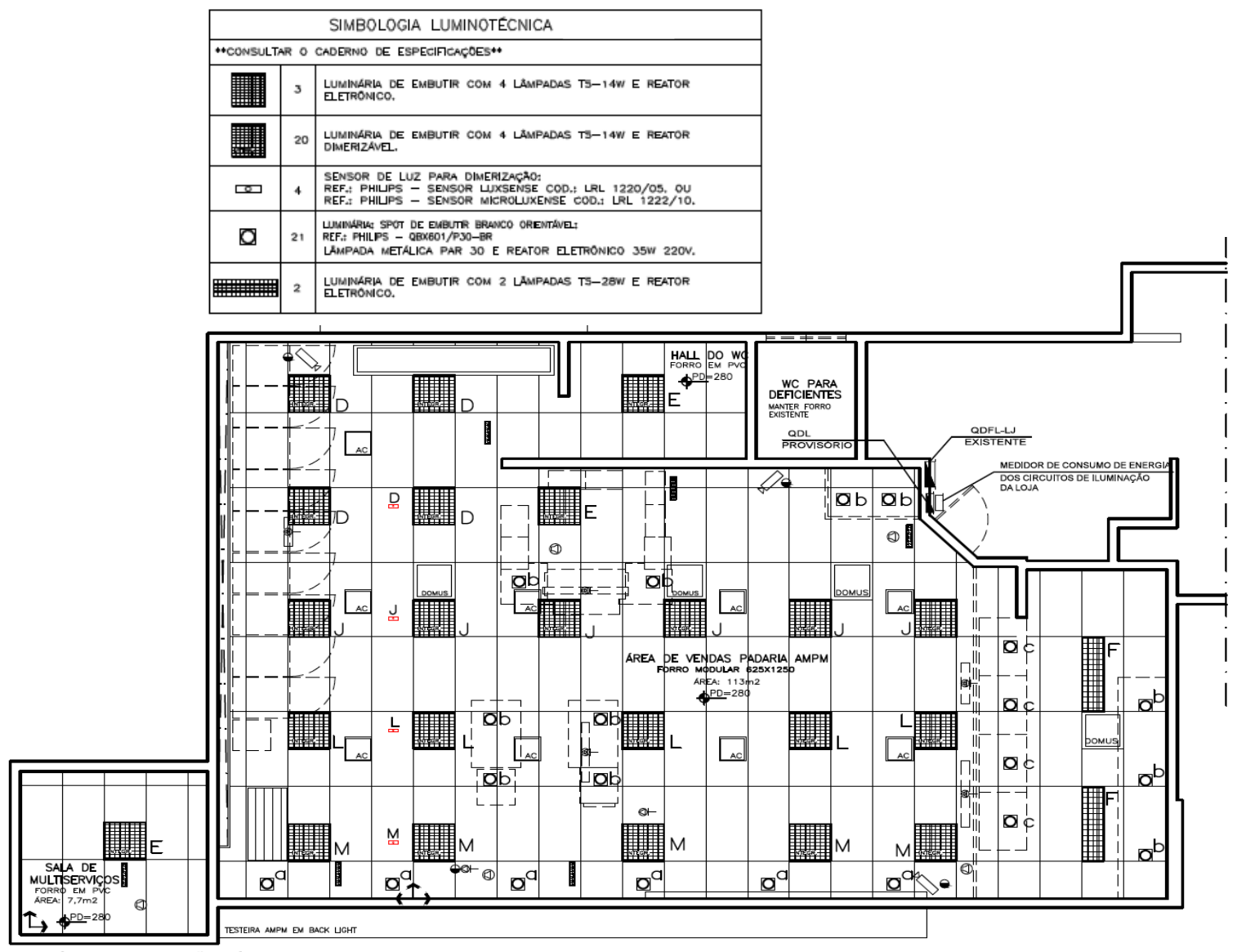

Fonte: Autores

\subsection{Situação 3 e 4 - Panel Flat em LED 40W + PAR 30 em LED 12W - sensores desligados (3) e ligados(4)}

Para a distribuição das luminárias no teto foi realizada a simulação com a iluminância de 400 lux pretendida, chegando-se ao quantitativo de 16 luminárias panel flat 40W e 24 PAR 30 LED 12W(Figura 4). Foram reinstalados 3 sensores, assinalados em vermelho (Figura 4), ligados a 3 grupos de seções de luminárias panel flat $(\mathrm{J} / \mathrm{L} / \mathrm{M})$. $\mathrm{O}$ quarto sensor ligado a seção $\mathrm{F}$ não foi alterado, pois na área de padaria foi mantido o padrão de 500lux. As luminárias pontuais não foram ligadas aos sensores pelo fato de não serem dimerizadas. 
Figura 4 - Planta de teto da situação 3 e 4 : panel flat 40W + PAR LED 12W com a marcação dos sensores

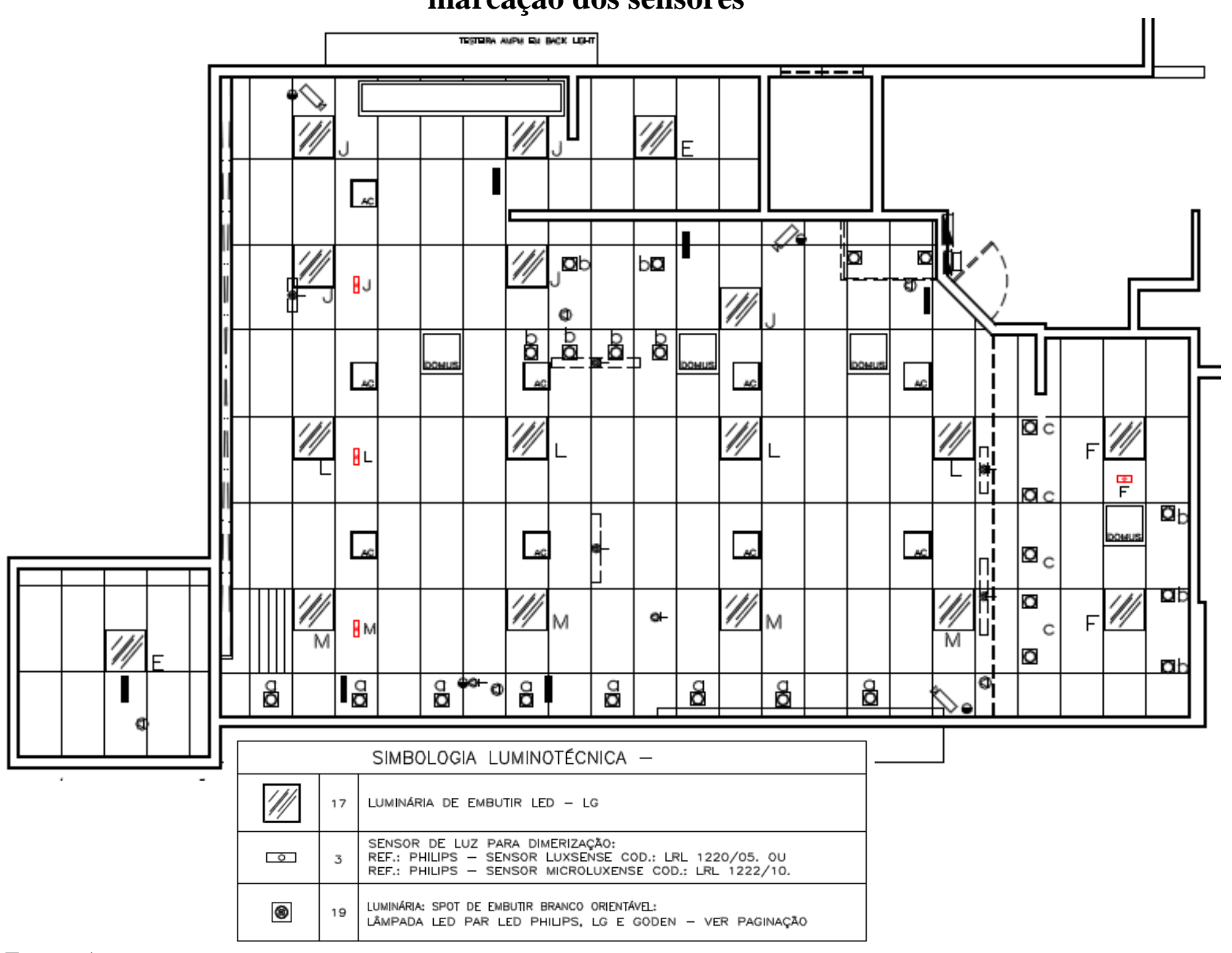

Fonte: Autores

\subsection{Simulações da situação 1 e 2}

Nesta etapa, foram realizadas duas simulações com o software AGI-32 com as fotometrias das respectivas luminárias. A simulação realizada com as lâmpadas fluorescentes T5 4x14W, considerando coeficiente de depreciação de 30\% encontrou média de iluminância de 406 lux. A simulação realizada com as lâmpadas fluorescentes T5 4x14W + vapor metálico PAR 30 de $35 \mathrm{~W}$, com o mesmo coeficiente de depreciação de 30\%, encontrou média de iluminância de 707 lux. Observou-se que a iluminação de destaque com as lâmpadas vapor metálico PAR 30 acrescentava grande contribuição em lux na média da loja.

\subsection{Medições da situação 1 e 2}

Para a realização das medições, a norma adotada foi a ABNT NBR ISO/CIE 8995-1 (2013). A primeira medição de iluminâncias foi realizada à noite, no dia 19/09/2013 às 19:00h, com os sensores desligados. O resultado encontrado foi de 647lux. A segunda medição foi realizada no dia 27/09/2013 as 12:30h, encontrando 821 lux, ainda com os sensores desligados com lâmpadas acesas. O céu estava parcialmente encoberto (Figura $5)$. 
Figura 5 - Foto do céu - medição: 27/09/2013 às 12:30h - sensores desligados com lâmpadas acesas

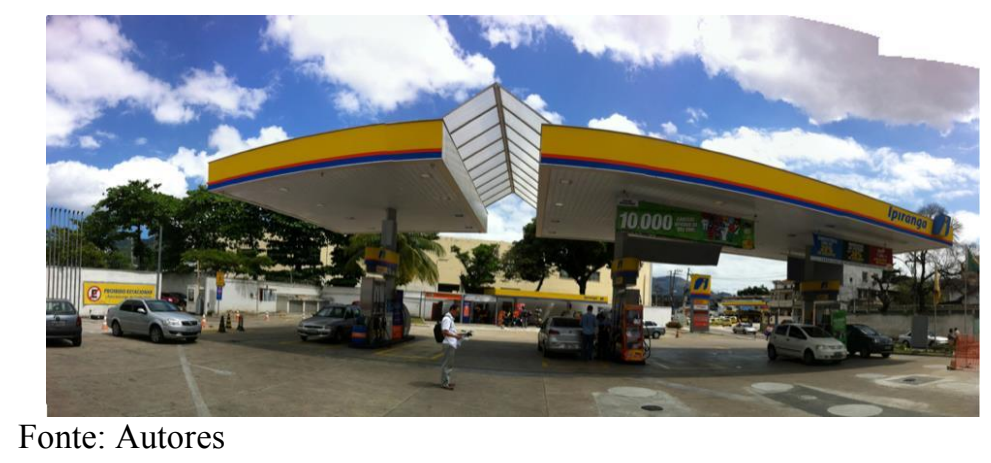

Para as medições de consumo com os sensores ligados, o ajuste foi realizado no dia 29/09/2013 conforme recomendação do fabricante Philips para 500lux. A terceira medição foi realizada no dia $03 / 10 / 2013$ às $12: 35$ h, encontrando 872 lux com os sensores ligados com lâmpadas acesas . O céu estava mais encoberto do que na medição anterior (Figura 6).

\section{Figura 6: Foto do céu - medição 03/10/2013 às 12:35 - sensores ligados com lâmpadas acesas}

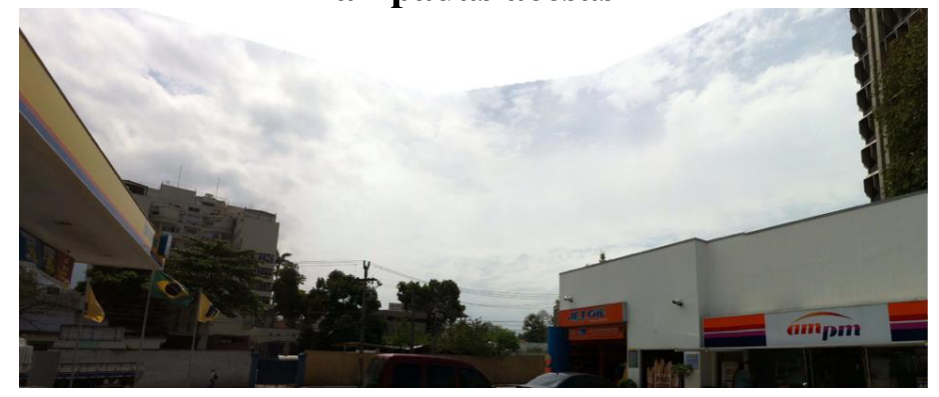

Fonte: Autores

\subsection{Simulações da situação 3 e 4}

Nesta etapa foi realizada simulação com o software AGI-32 com as fotometrias das respectivas luminárias. A simulação foi realizada com 17 luminárias LG LED Flat Light de $40 \mathrm{~W}$ e 24 PAR LED da Philips de 12W. A média de iluminância encontrada foi de 444 lux com DPI de $9 \mathrm{~W} / \mathrm{m} 2$.

\subsection{Medições da situação 3 e 4}

A primeira medição de iluminâncias foi realizada à noite, no dia 11/10/2013 às 20:00h, com os sensores desligados. O resultado encontrado foi de 486 lux. A segunda medição foi realizada no dia 15/10/2013 às 13:00h, com os sensores ligados com lâmpadas acesas, e céu parcialmente encoberto (Figura 7). O resultado encontrado foi de 466 lux.

$\mathrm{Na}$ noite anterior a medição do dia 15/10/2013 foi realizada a regulagem dos sensores. O primeiro sensor regulado foi o que comanda a seção $\mathrm{J}$, o mais distante da vitrine da loja (Figura 4). O luxímetro foi colocado a $0,75 \mathrm{~cm}$ do piso, e imediatamente embaixo da luminária. Quando isto não foi possível, manteve-se um raio de $0,5 \mathrm{~m}$ ao redor do eixo vertical da luminária. O sensor foi ajustado para 400 lux. O mesmo procedimento foi realizado para os sensores que controlam as seções L e M. 
Figura 7: Foto do céu - medição 15/10/2013 às 13:49 - sensores ligados com lâmpadas acesas

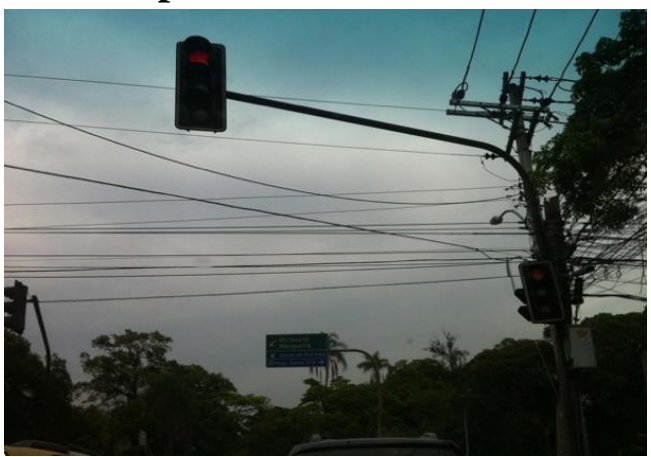

Fonte: Autores
Figura 8: Foto do céu - medição 13/03/2014, às 14:32 - sensores ligados com lâmpadas acesas

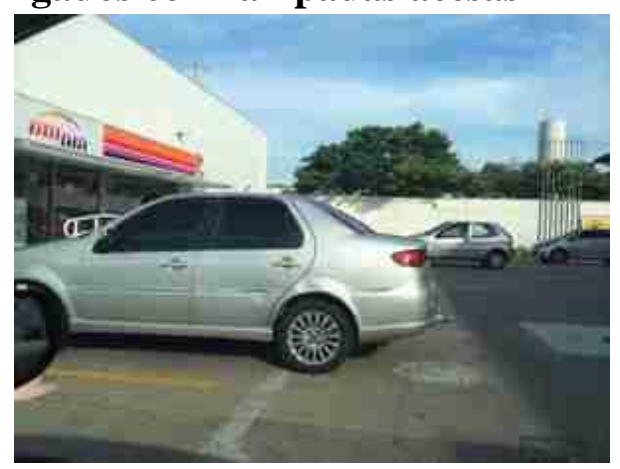

Fonte: Autores

A terceira medição foi realizada na noite do dia 14/01/2014 às 20:00h, com os sensores ligados, com o objetivo de verificar se a regulagem dos sensores estava coerente com a iluminância proposta de 400lux. A média da iluminância encontrada foi de 409 lux. Foi realizada uma nova medição no dia 13/03/2014 às 14:32 para revisar os resultados durante o dia com os sensores ligados com lâmpadas acesas, com céu mais limpo (Figura 8), pois na primeira medição do dia 15/10/2013 (Figura 7) o céu se encontrava muito encoberto. O resultado dessa medição foi de 696 lux.

\subsection{Medições de consumo}

Para as quatro situações propostas de medição de consumo, o Quadro 1 e a Figura 9 mostram a significativa redução no consumo de energia elétrica que ocorreu na mudança da tecnologia de sistema de iluminação, que passou a adotar as luminárias LED em substituição às luminárias com lâmpadas T5, somada a redução de iluminância da loja. Comparando a situação 1 sem os sensores, com a situação 3 também sem sensores, observa-se que houve uma redução de aproximadamente 57\% no consumo de energia com ilumnação.

\section{Figura 9: Gráfico final das medições de consumo de energia elétrica}

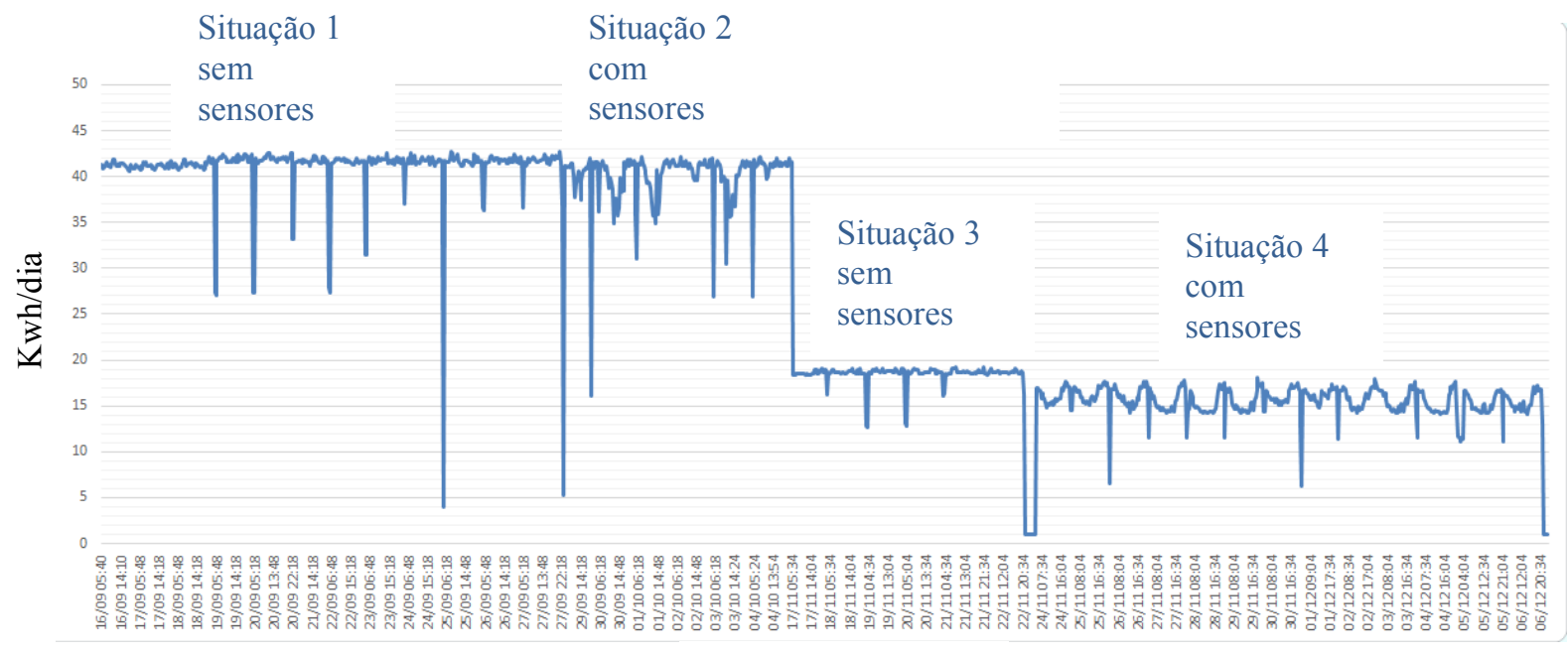

Data/Horário

Fonte: Autores 
Quadro 1: Resumo das medições de consumo de energia elétrica

\begin{tabular}{|c|c|c|c|c|}
\hline & $\begin{array}{c}\text { Situação 1 } \\
\text { T5 sem sensor }\end{array}$ & $\begin{array}{c}\text { Situação 2 } \\
\text { T5 com sensor }\end{array}$ & $\begin{array}{c}\text { Situação 3 } \\
\text { LED sem sensor }\end{array}$ & $\begin{array}{c}\text { Situação 4 } \\
\text { LED com sensor }\end{array}$ \\
\hline $\begin{array}{c}\text { Consumo kWh/dia } \\
\text { médio }\end{array}$ & 41,43 & 39,39 & 17,85 & 14,47 \\
\hline $\begin{array}{c}\text { Consumo mensal medio } \\
\mathrm{kWh} / \mathrm{mês}\end{array}$ & $1.242,90$ & $1.181,70$ & 535,50 & 434,10 \\
\hline
\end{tabular}

O emprego do sensor para controle da iluminação avaliado na situação 4, com LEDs, registra uma redução mais significativa em relação a redução registrada na situação 2 com T5, em virtude da redução do nível de iluminância da loja que passou de 500 lux (valor padrão do sensor Philips) para 400 lux, conforme orientação do projeto luminotécnico. Enquanto o uso do sensor no sistema de iluminação na situação 2 registra uma redução no consumo de energia elétrica de aproximadamente 5\%, o uso do sensor utilizado no sistema de iluminação na situação 4 aponta uma redução de aproximadamente $19 \%$ no consumo de energia elétrica.

\section{DISCUSSÃO}

O Quadro 2 mostra um resumo comparando todas as situações medidas.

Quadro 2: Resultado das medições de iluminâncias na loja am/pm

\begin{tabular}{|c|c|c|c|c|c|c|c|}
\hline \multicolumn{7}{|c|}{ RESUMO DAS MEDIÇÕES DE ILUMINÂNCIA NA LOJA MATRIZ AMPM IPIRANGA } \\
\hline LUMINÁRIA & $\begin{array}{r}\text { STUAÇAO 1 e 2 - FLUORESCENTE } \\
\text { T5 4X14W + VM 35W }\end{array}$ & \multicolumn{5}{c|}{ STUAÇAO 3 e 4 - PANEL FLAT 40W + PAR LED } \\
\hline DIA & $19 / 09 / 2013$ & $27 / 09 / 2013$ & $03 / 10 / 2013$ & $11 / 10 / 2013$ & $15 / 10 / 2013$ & $14 / 01 / 2014$ & $13 / 03 / 2014$ \\
\hline HORA & $19: 00$ & $12: 30$ & $12: 35$ & $20: 00$ & $13: 00$ & $20: 00$ & $14: 32$ \\
\hline TURNO & NOITE & DIA & DIA & NOTE & DIA & NOITE & DIA \\
\hline SENSOR & S/ SENSOR & S/ SENSOR & C/ SENSOR & S/ SENSOR & C/ SENSOR & C/ SENSOR & C/ SENSOR \\
\hline MÍN/MÉD & 0.4 & 0.44 & 0.53 & 0.6 & 0.47 & 0.46 & 0.49 \\
\hline MÍN/MÁX & 0.13 & 0.18 & 0.16 & 0.29 & 0.2 & 0.39 & 0.2 \\
\hline MÉDIA & 647 & 821 & 872 & 486 & 466 & 409 & 696 \\
\hline
\end{tabular}

Com relação as medições de iluminância da situação 1 sem sensor, a realizada no dia 19/09/2013 mostra um valor médio de 647 lux. Ao se comparar com o resultado da simulação do software AGI-32, com coeficiente de depreciação de $30 \%$, que apresentou média de 707 lux (item 3.3), observa-se que os valores estão muito próximos, e que essa diferença de 50 lux pode se atribuir ao fator de depreciação do sistema ser maior do que os 30\% estipulados para a simulação. Observou-se que muitas lâmpadas, principalmente as fluorescentes na ocasião da medição, apresentavam-se com depreciação visível na intensidade do fluxo luminoso.

Na segunda medição da situação 1 , sem sensor, realizada no dia 27/09/2013 às 12:30h, o céu estava parcialmente encoberto (Figura 5), e o resultado encontrado foi de 821 lux. Com os sensores ligados, e regulados para 500 lux, conforme indicação dada pelo fabricante, foi feita uma nova medição no dia 03/10/2013 às 12:35h, e o resultado ficou em 872 lux, muito próximo da medição anterior sem sensor. O céu estava bem mais encoberto (Figura 6) do que na medição anterior. Entretanto, a medição de energia mostrou que, durante a semana na qual o sensor estava ligado, houve de fato uma pequena redução de 5\% no consumo (Figura 9 e Quadro 1).

O grande ganho deu-se com a modificação para a situação 3 e 4, devido a mudança das fontes para LEDs em toda a loja, somada a diminuição da iluminância para 400 lux. Ao 
se analisar o gráfico de consumo (Figura 9 e Quadro 1), constatou-se que houve considerada economia em kWh. Na medição realizada no dia 11/10/2013 da situação 3, à noite com os sensores desligados, chegou-se à iluminância de 486 lux. Comparando com a medição da noite do dia 14/01/2014 às 19:00h, onde os sensores já haviam sido ligados e regulados para 400 lux, chegou-se ao valor de 409 lux, mostrando que houve de fato diminuição da iluminância e de consumo com o ajuste dos sensores (Quadro 2).

A medição feita no dia 15/10/2013 às 13:00, com os sensores ligados e regulados para 400 lux, apontou um resultado de 466 lux, muito próximo da medição noturna com sensores do dia 14/01/2014 (409 lux). Isso pode ser atribuído ao céu neste dia (Figura 7) que estava bastante encoberto. Esta medição foi refeita no dia 13/03/2014 às 14:30, alcançando o resultado de 696 lux (Figura 8). Esta medição indica a influência do tipo de céu na ocasião das medições, e também a época do ano em que foram realizadas, apontando um acréscimo de luz natural de aproximadamente 200 lux. Ressalta-se a necessidade de um maior aprofundamento nos registros obtidos em estações metereológicas locais para complementação dessa informação.

A avaliação do uso de sensores se mostra positiva em relação a redução de consumo de energia. Para a situação 2 , a redução no consumo de energia elétrica foi de aproximadamente 5\%. O estudo mostra que a regulagem dos sensores, de 500lux para 400lux significou, na situação 4, cerca de 19\% de economia. Sabe-se que influenciam também nestes resultados outras variáveis, que não somente a alteração da iluminância, tais como: diferentes tipologias de plantas e arranjos de luminárias, latitude, clima, tipo de céu, quantidade de aberturas e orientação solar.

\section{CONCLUSÃO}

Em conclusão, o estudo mostrou redução de aproximadamente 57\% no consumo de energia elétrica, ocorrida com a mudança da tecnologia para LED, associada a nova proposta de iluminância, sem o uso de sensores. Outra importante constatação foi a grande diminuição do DPI (lighting power density) para $9 \mathrm{~W} / \mathrm{m}^{2}$, superando o esperado pela ASHRAE 90-1, que para a lojas de conveniências, deveria ser de no máximo 18,10 $\mathrm{W} / \mathrm{m}^{2}$.

Com relação a utilização de sensores de controle, que integram a iluminação natural à artificial, o estudo mostra que de fato estes contribuem para a diferenciação do consumo. Constatou-se que a adequação da iluminância foi importante nesse estudo porém, o padrão analógico apresenta muita dificuldade de regulagem quando se deseja iluminâncias diferentes de 500 lux (padrão dos sensores). A solução digital, que permite ao usuário a programação e dimerização sistemática para cada luminária ou grupamento de luminárias, apresenta-se mais eficiente, embora mais dispendiosa.

Com base nesses resultados, a iluminação com LEDs tem sido gradualmente implementada, sempre após estudo de viabilidade econômica, como um ítem de grande contribuição para a eficiência energética das lojas.

\section{REFERÊNCIAS BIBLIOGRÁFICAS}

ASHRAE - Standard 90.1: Energy Standard for Buildings Except Low-Rise Residential Buildings - Lighting and Power Requirements, 2007.

ASSOCIAÇÃO BRASILEIRA DE NORMAS TÉCNICAS (ABNT). NBR ISO/CIE 8995-1: Lighting of work places Part 1: Indoor, 2013.

IES - The Lighting Handbook - 10th Edition: Reference and Aplication, 2011. 